\title{
Combined transconjunctival-eyebrow approach providing minimally invasive access to all orbital quadrants
}

\author{
Technical note
}

\author{
Andrei Koerbel, M.D., ${ }^{1}$ Veralucia R. Ferreira, M.D., ${ }^{2}$ And André Kiss, M.D. ${ }^{1}$ \\ Departments of ${ }^{1}$ Neurosurgery and ${ }^{2}$ Ophthalmology, Neuroscience Institute, Joinville, Santa Catarina, \\ Brazil
}

\begin{abstract}
$\checkmark$ Surgical approaches to treat orbital disease should provide a good exposure of intraorbital anatomical structures, allow their functional preservation, and provide good cosmetic results. The authors describe a minimally invasive, combined transconjunctival-eyebrow approach to all orbital quadrants in a step-wise manner. The indications, advantages, and limitations of the technique are highlighted.

A transconjunctival approach via the postseptal area is described. It allows exposure of the medial, inferior, and lateral parts of the orbit. Depending on the orbital space to be exposed, a lateral or a medial eyebrow incision is then made. The eyebrow and the conjunctival incisions are connected by subperiosteal dissection. This combined access provides exposure to all intraconal muscles and to the superior, medial, lateral, and inferior portions of the optic nerve.

The combined transconjunctival-eyebrow approach provides an excellent orbital exposure, with minimal damage to the circumjacent structures. It requires less operative time than other approaches and yields good cosmetic results. Intracranial or intrafacial tumor extension and tumors located purely in the orbital apex are limitations for the use of this technique. (DOI: 10.3171/FOC-07/11/E10)
\end{abstract}

KeY WORDS - minimally invasive surgery - orbit • transconjuctival-eyebrow approach • tumor

$\mathrm{T}$

HE orbit is a small compartment containing very delicate structures that are essential for the function of vision. Approaches to treat orbital diseases should provide a good exposure of anatomical structures and allow preservation of their function, such as ocular motility and visual acuity. Additionally, in the cases of exophthalmos or ocular deviation, surgery should maintain or improve a patient's physiognomical appearance.

Several approaches to the intraorbital space have been described in the literature. ${ }^{3,5,8,12}$ Most require bone removal through orbitotomies and/or craniotomies.

More recently, transconjunctival approaches have been reported as suitable for the removal of tumors located in the inferomedial, basal, and lateral region of the orbit., ${ }^{4,7}$ Moreover, they preclude the need for bone resection and subsequent reconstruction, offering less extensive and traumatic approaches. ${ }^{4}$

Abbreviation used in this paper: $\mathrm{MR}=$ magnetic resonance.
In an attempt to maintain this philosophy and to provide an increased operative exposure, we describe a transconjunctival approach combined with an eyebrow incision in which bone removal or intraconal muscle sectioning are not required. It provides access to the superior, medial, lateral, and inferior quadrants of the orbit, depending on the extension of the conjunctival and eyebrow incisions. Thus, complete exposure and resection of very large intraorbital lesions are feasible with minimal invasiveness and reduced morbidity.

\section{Surgical Technique}

The patient is positioned supine after induction of general anesthesia. According to the location of the lesion, the conjunctival and the eyebrow incision are extended more laterally or medially.

The transconjunctival approach is initiated by making an 


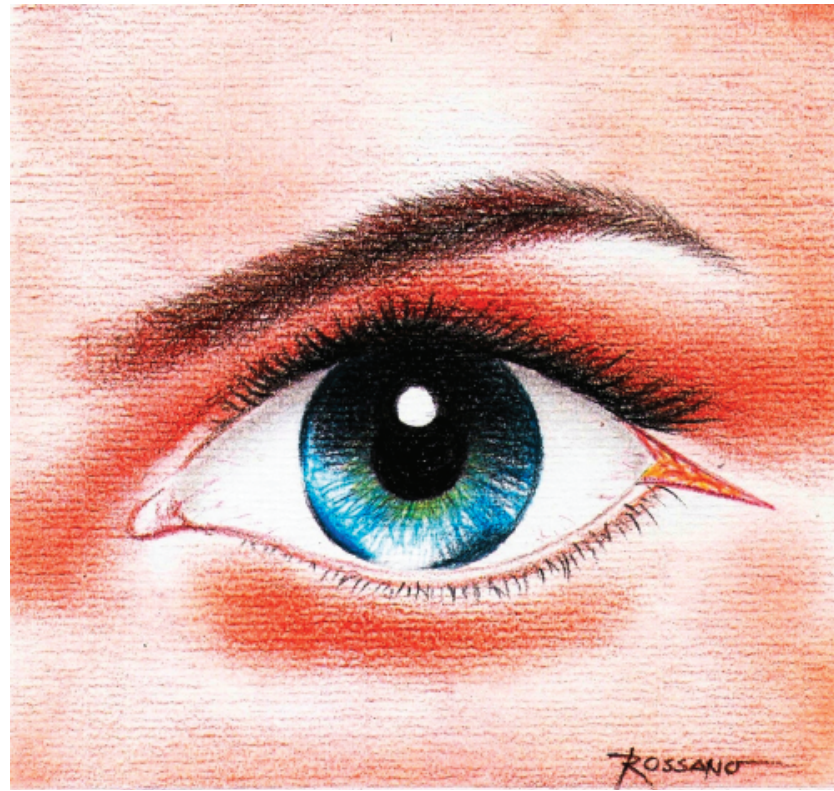

FIG. 1. Artist's drawing. The transconjunctival approach is initiated by making an incision at the canthal angle and following in the canthal skin crease.

incision at the canthal angle and following in the canthal skin crease (Fig. 1). After canthotomy, the connections of the lateral canthal tendon to the orbital septum and periorbita are released. ${ }^{16}$ The eyelids are spread apart and the inferior fornix is exposed. Beginning laterally, the conjunctiva is incised in the inferior fornix and extended medially (Fig. 2). The lower lid is divided into preseptal, septal, and postseptal regions. ${ }^{6}$ The preseptal region includes skin, subcutaneous tissue, orbicularis oculi muscle and suborbicularis fascia, and the septal region contains the orbital septum and tarsal plate. ${ }^{6}$ The postseptal area includes the

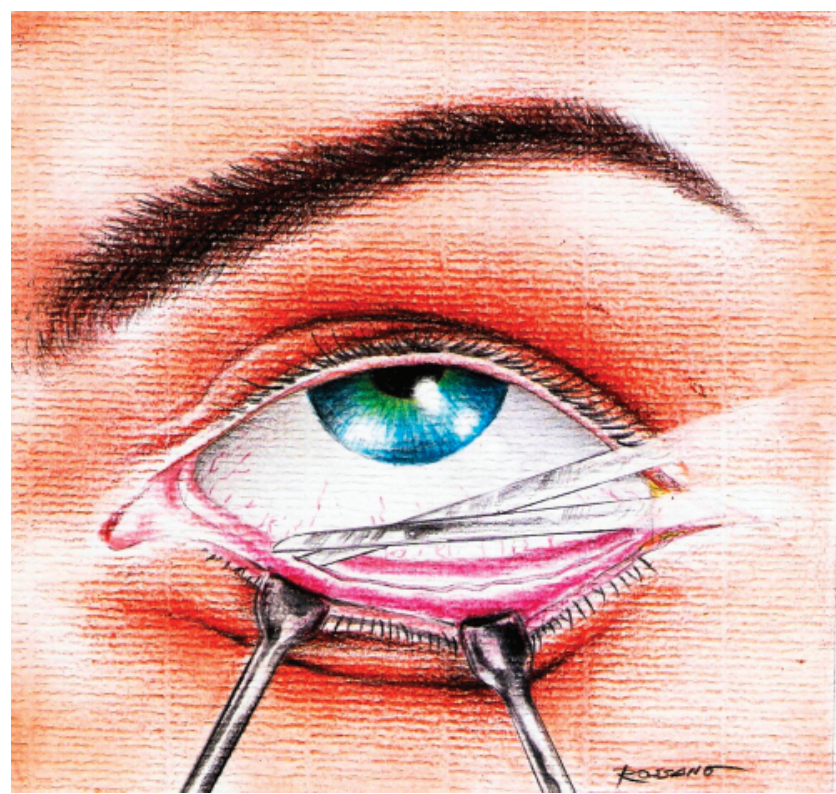

FIG. 2. Beginning laterally, the conjunctiva is incised in the inferior fornix and extended medially.

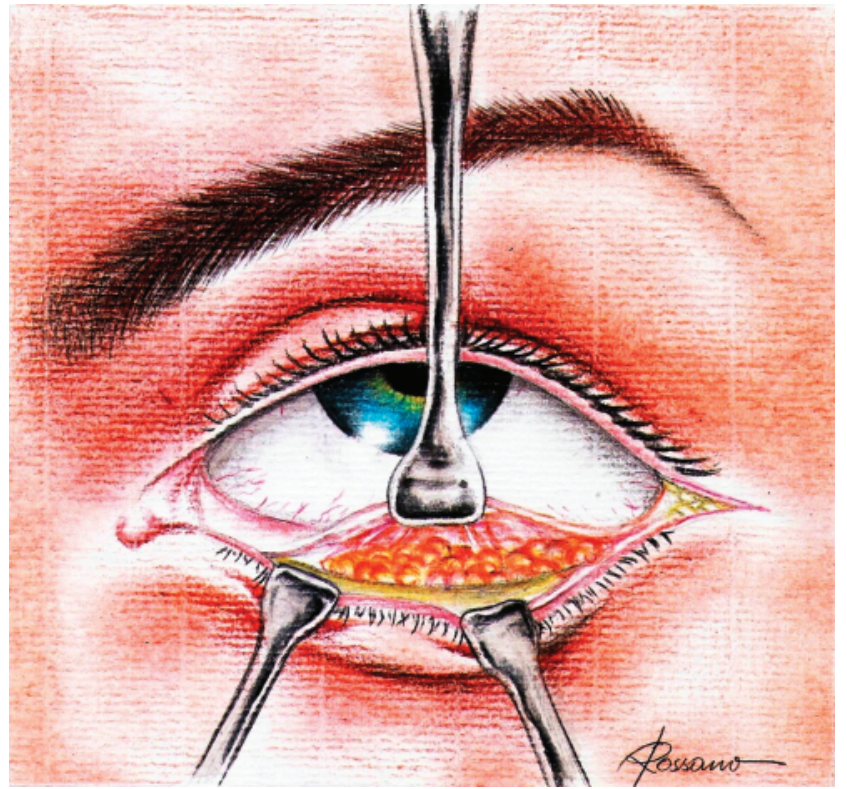

FIG. 3. After incision of the periorbita, the orbital contents are visualized, and access to the intraconal space is achieved after retracting the ocular globe superiorly.

palpebral conjunctiva and the extension of the orbital fat. ${ }^{6}$ Thus, the postseptal route is preferred, because preseptal access may lead to ectropion due to septal damage. ${ }^{6}$ The capsulopalpebral fascia, a fibrous white band, is exposed over the inferior orbital rim. ${ }^{12}$ Opening the capsulopalpebral fascia, the periorbita is seen. Following its incision, the orbital contents are visualized, and access to the intraconal space is achieved after retracting the ocular globe superiorly (Fig. 3). The microscope is then brought on the operative field to allow a better visualization of the muscular and nervous structures. At this point, the basal, the inferomedial, and the inferolateral regions of the orbit can be accessed. The lateral rectus muscle, laterally, and the medial rectus muscle, medially, are the limits of the exposure. The inferior, medial, and lateral portions of the optic nerve can be fully visualized.

According to the region of the orbit to be approached, an eyebrow incision is made medial or lateral to the supraorbital nerve. The incision's extension varies from 2.5 to 4 $\mathrm{cm}$, depending on the required exposure. The orbicularis oculi muscle is incised, and the superior orbital rim is exposed. The eyebrow and the conjunctival incisions are connected by a subperiosteal dissection. A self-retaining retractor is used to spread the soft tissue apart. Gentle dissection allows separation of the periorbita from the superior orbital rim and orbital roof, as well as medial or lateral orbital wall dissection, according to the skin incision. After opening the periorbita, the globe is then retracted inferiorly (Fig. 4). The superior rectus-levator muscle complex, the lacrimal gland, and the superior, lateral, and medial portions of the optic nerve may be fully exposed. Depending on the skin incision, the lateral or medial rectus muscle may be also exposed.

Following tumor resection, the orbicularis oculi muscle is approximated and the skin is closed using interrupted sutures or intradermic suture. 


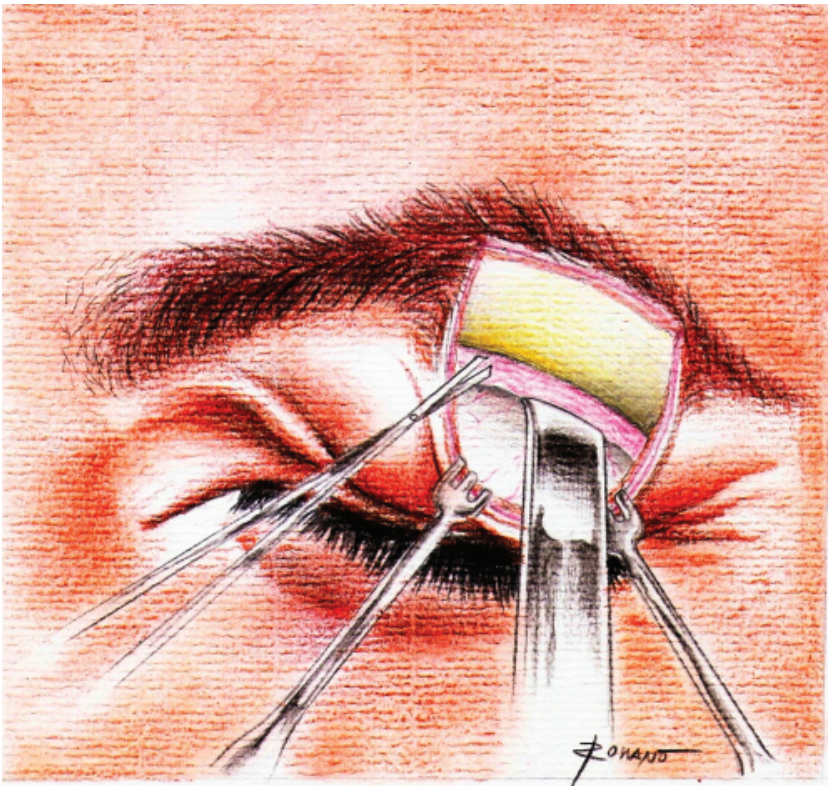

FIG. 4. According to the region of the orbit to be approached, an eyebrow incision is made medial or lateral to the supraorbital nerve. A lateral eyebrow incision is shown in the illustration. After opening the periorbita, the ocular globe is then retracted inferiorly and the superior intraorbital contents are accessed.

Regarding the transconjunctival approach, the periorbita and capsulopalpebral fascia do not need to be closed. The conjunctiva is closed with a running 8-0 absorbable suture. The canthal tendon is fixed to the orbital rim with Prolene. The canthal crease skin may be closed using interrupted sutures or intradermic suture.

\section{Illustrative Case}

This 40-year-old woman presented with a 6-month history of increasing left-eye proptosis associated with decrease of visual acuity. Computed tomography scanning revealed a very large, well-circumscribed tumor in the left orbit. Magnetic resonance imaging demonstrated a large, well-delimitated enhancing mass occupying the superior, lateral, inferior, and central portions of the orbit. The optic nerve was severely compressed by the tumor (Fig. 5).

A combined transconjunctival-eyebrow approach was planned to remove the tumor. A lateral eyebrow incision was made first, and the tumor was partially shrunken using bipolar forceps. Afterward the transconjunctival approach was performed, and the inferior portion of the mass was then reached. The tumor was significantly reduced in size by step-wise shrinking. The mass could be totally removed while preserving all nerves and muscles.

The postoperative period was uneventful and there were no postoperative deficits. The patient's visual acuity remained stable. Immediate postoperative MR imaging demonstrated radical tumor resection (Fig. 6). The pathological report indicated the specimen was a cavernous hemangioma.

\section{Discussion}

Most approaches used to remove orbital tumors typically include bone removal (orbitotomies) with or without craniotomies.

In 1889, Krönlein ${ }^{8}$ first described the lateral orbitotomy, which became the preferred approach in the pre-computed tomography era for resecting orbital lesions. McNab and Wright $^{13}$ performed surgery in 85 cases of cavernous hemangiomas and used a lateral orbitotomy in 71 of them. Three of their patients suffered from visual loss postoperatively.

Transcranial approaches have been preferentially used by neurosurgeons. In the pre-computed tomography era, Dandy $^{3}$ resected 24 orbital tumors via a subfrontal transcranial approach. Because the tumor's exact location was in question, many other authors have used transcranial access routes. ${ }^{11}$ Moreover, the belief existed that excessive orbital edema could occur after surgery and transcranial orbital decompression could save the visual acuity of the patient in this case. ${ }^{4}$

Recently, Missori et al. ${ }^{14}$ undertook surgery in 23 patients with cavernous hemangiomas by first performing transcranial orbitotomies. Three patients presented with ptosis after surgery and in 8 patients visual acuity worsened after the operation. In another study involving 13 patients in whom
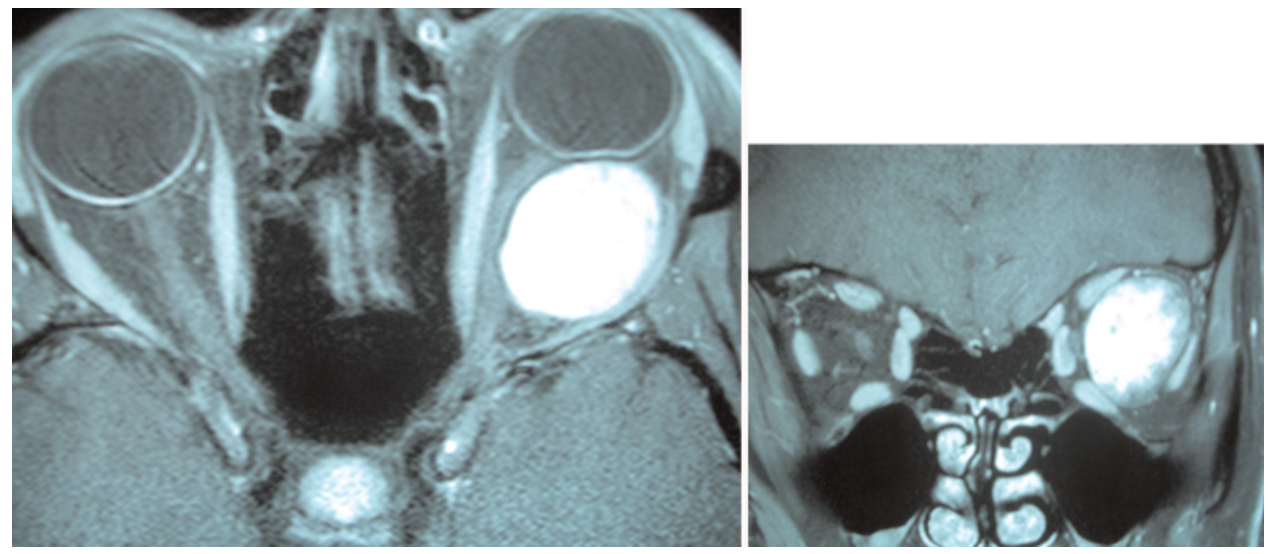

FIG. 5. Axial (left) and coronal (right) T1-weighted Gd-enhanced MR images showing an enhancing, very large mass involving the superior, lateral, inferior, and central portions of the orbit. The optic nerve was severely compressed by the tumor. 


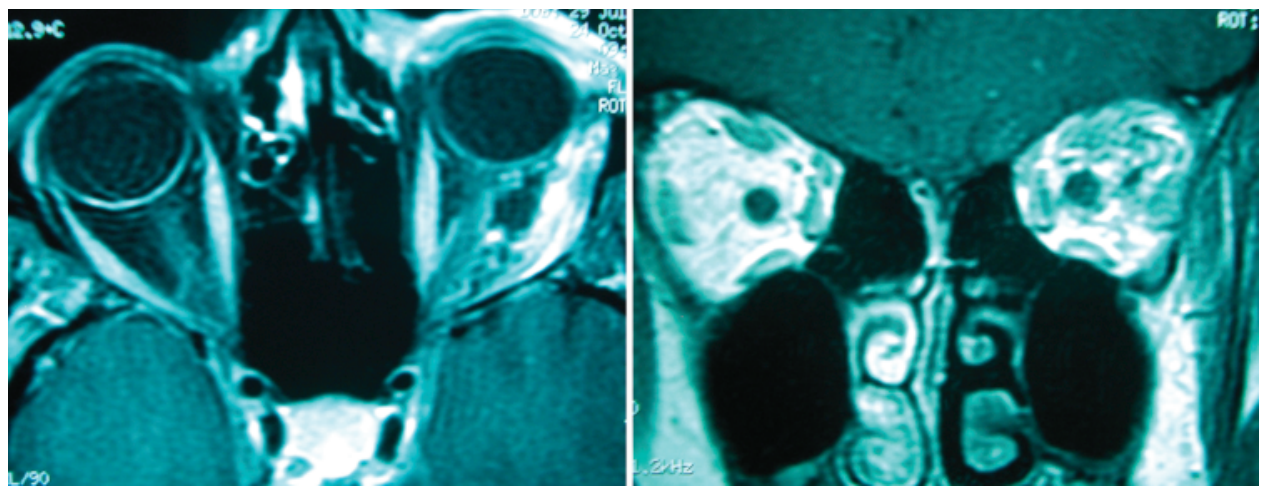

FIG. 5. Postoperative axial (left) and coronal (right) MR images demonstrating complete removal of the cavernous hemangioma.

surgery was undertaken through a frontal craniotomy, one patient experienced visual loss after the procedure. ${ }^{1}$

More recently, transconjunctival approaches have been used in the treatment of intraorbital tumors, and the rates of morbidity and no mortality have been very low. ${ }^{9,10}$ Kiratli et al. ${ }^{7}$ reported on 24 patients in whom a transconjunctival approach was used to excise intraconal cavernous hemangiomas. In the postoperative period, vision improved in 8 patients and remained stable in the others. No patient had visual acuity aggravation.?

Transconjunctival approaches have also been described for minimally invasive orbital decompression and for the treatment of orbital fractures. ${ }^{2,6,12}$

Transconjunctival approaches offer several advantages to the patient and surgeon. The approach produces no visible scar and reduces the incidence of enophthalmos because the orbital wall is not removed. Thus, the patient may have his proptosis corrected after tumor resection without fat herniating through the orbitotomies and consequent enophthalmos (based on the Hertel curve). ${ }^{15}$

As no bone removal is required, this is a minimally invasive procedure. Thus, trauma to the surrounding orbital structures is reduced and only a short operative time is required.

However, there are limitations concerning its applicability: 1) Lesions located purely in the orbital apex cannot be adequately exposed by this approach. The more anteriorly the anterior portion of the tumor is, the better the achieved exposure. 2) Lesions in the medial, inferior, and lateral quadrants of the orbit are well reached by this via this exposure, whereas lesions situated in the superior orbital portion are not adequately visualized.

Therefore, a combined access should be considered in selected cases. Depending on the location of the eyebrow incision, the superomedial or-lateral (or both) parts of the orbit can be widely reached. All orbital quadrants can be reached easily with reduced operative time.

The only major surgical limitations for this combined approach are tumors that extend extraorbitally (intracranial or-facial) and tumors located in the orbital apex.

The anular tendon, which arises from the rectus muscles, involves part of the superior orbital fissure and part of the optic foramen. The lacrimal nerve, the trochlear nerve, and the frontal nerve pass through the superior orbital fissure outside of the anular tendon. The oculomotor nerve, the abducent nerve, and other small nerves penetrate into the orbit through the oculomotor foramen. The orbital apex includes this transition area between the intracranial andorbital space, which can only be entirely exposed by opening the optic canal and the superior orbital fissure via a transcranial approach. The orbital space just anterior to the anular tendon can be exposed through a transconjunctival approach.

In summary, even very large orbital tumors can be resected after taking a minimally invasive combined transconjunctival-eyebrow approach. In using this technique, there are no risks of cerebrospinal fluid leakage or significant blood loss during the procedure, no bone removal or reconstruction is required, and the patient recovers satisfactorily in a short postoperative time. The access allows an excellent exposure of the orbital structures and the angle of view is very favorable for the surgeon. Because there is no fat herniation in the operative field, the surgeon has an increased facility for spatial orientation. The cosmetic results are very satisfactory, because the incisions are small and hidden, the incidence of enophthalmos is low, and there is no temporal muscle atrophy.

\section{References ]}

1. Acciarri N, Giulioni M, Padovani R, Gaist G, Pozzati E, Acciarri R: Orbital cavernous angiomas: surgical experience on a series of 13 cases. J Neurosurg Sci 39:203-209, 1995

2. Ben Simon GJ, Schwarcz RM, Mansury AM, Wang L, McCann JD, Goldberg RA: Minimally invasive orbital decompression: local anesthesia and hand-carved bone. Arch Ophthalmol 123: 1671-1675, 2005

3. Dandy W: Results following the transcranial operative attack on orbital tumors. Arch Ophthalmol 25:191-216, 1941

4. Hassler W, Schaller C, Farghaly F, Rohde W: Transconjunctival approach to a large cavernoma of the orbit. Neurosurgery 34: 859-862, 1994

5. Housepian E: Microsurgical anatomy of the orbital apex and principles of transcranial orbital exploration. Clin Neurosurg 25: 556-573, 1978

6. Ilankovan V: Transconjunctival approach to the infraorbital region: a cadaveric and clinical study. Br J Oral Maxillofac Surg 29:169-172, 1991

7. Kiratli H, Bulur B, Bilgic S: Transconjunctival approach for retrobulbar intraconal orbital cavernous hemangiomas. Orbital surgeon's perspective. Surg Neurol 64:71-74, 2005 
8. Krönlein R: Zur Pathologie und operativen Behandlung der Dermoidzysten der Orbita. Beitr Klin Chir 4:149-163, 1889

9. Lazar M, Rosen N, Geyer O, Godel V: A transconjunctival cryosurgical approach for intraorbital tumors. Aust N Z J Ophthalmol 13:417-420, 1985

10. Loewenstein A, Geyer O, Lazar M: Cavernous haemangioma of the orbit: treatment by transconjunctival cryoextraction. Eye 7: 597-598, 1993

11. Love J, Bryar G: Transcranial extirpation of orbital tumors. $\mathbf{T r}$ Am Acad Ophthalmol Otol 70:620-626, 1966

12. Maroon JC, Kennerdell JS: Surgical approaches to the orbit. Indications and techniques. J Neurosurg 60:1226-1235, 1984

13. McNab AA, Wright JE: Cavernous hemangiomas of the orbit. Aust N Z J Ophthalmol 17:337-345, 1989

14. Missori P, Tarantino R, Delfini R, Lunardi P, Cantore G: Surgical management of orbital cavernous angiomas: prognosis for visual function after removal. Neurosurgery 35:34-38, 1994

15. Neuschwander TB, Chang EL, Sadun AA: Hertel curve: orbital volume increment and proptosis in a cadaver model. Ophthal Plast Reconstr Surg 21:431-434, 2005

16. Shore JW: The fornix approach to the inferior orbit. Adv Ophthal Plast ReconstrSurg 6:377-385, 1987

Manuscript submitted August 13, 2007.

Accepted September 27, 2007.

Address correspondence to: Andrei Koerbel, M.D., Department of Neurosurgery, Neuroscience Institute, Placido Olimpio de Oliveira Street, 1244, CEP 89202-451, Joinville, Santa Catarina, Brazil. email: andrei@apmelo.com.br. 